\title{
Prevalence of Peptic Ulcer Disease in Asymptomatic Subjects Referring to the Check-Up Clinic of Razavi Hospital from 2006 - 2011
}

\author{
Ali Abdolhosseini ${ }^{1}$; Kambiz Totonchi Sarraf ${ }^{1}$; Sarah Tayebi ${ }^{1}$; Azra Izanloo ${ }^{1,{ }^{*}}$; Najme Jafari \\ Moghadam ${ }^{1}$ \\ ${ }^{1}$ Education and Research Department, Razavi Hospital, Mashhad, IR Iran \\ ${ }^{*}$ Corresponding author: Azra Izanloo, Education and Research Department, Razavi Hospital, Mashhad, IR Iran. Tel: +98-5116004359, Fax: +98-5116004408, E-mail: a.izanloo@yahoo. \\ com
}

Received: October 22, 2013; Revised: November 30, 2013; Accepted: December 9, 2013

\begin{abstract}
Background: Life style change is considered as a factor affecting on the increasing number of subjects with peptic ulcer disease (PUD). Helicobacter pylori (H.pylori) infection is regarded as a risk factor for the mentioned disease.

Objectives:This study aims to analyze the prevalence of PUD in asymptomatic subjects referring to the check-up clinic of Razavi Hospital and the relation between PUD and $\mathrm{H}$. pylori.

Materials and Methods: 499 asymptomatic subjects who had referred to the Check-Up Clinic of Razavi Hospital from 2006 till 2011 included in this study. Their data including: age, sex, endoscopy report and rapid urease test (RUT) were evaluated and the results of 260 Biopsy were analyzed by SPSS.

Results: The mean age of the subjects was 52 which included 73.1\% males and $26.9 \%$ females. Positive H. Pylori were reported in $75.2 \%$ of males and $24.8 \%$ of females. Endoscopy results revealed $57.2 \%$ subjects with PUD from which $23 \%$ were seen with erosion, $11.6 \%$ with gastritis, $11.4 \%$ with ulcer, $8 \%$ with erythema, $1.6 \%$ with sub epithelial hemorrhage, $0.8 \%$ with deodenitis and $0.8 \%$ with gastroduodenitis. In $72 \%$ of subjects that their endoscopy showed PUD and in 38\% of the subjects with normal endoscopy, positive RUT was reported. There was a significant relation between PUD observed in endoscopy and RUT ( $\mathrm{P}=0.001)$. In $89.6 \%$ of subjects who had PUD in biopsy, $57.7 \%$ subjects had gastritis with positive $H$. pylori and $13.8 \%$ subjects had gastritis without $\mathrm{H}$. Pylori. There was a significant relation between gastritis incidence based on biopsy and RUT $(\mathrm{P}=0.001)$.

Conclusions: The prevalence of PUD is more common in asymptomatic males than females. Regarding the high prevalence of PUD and $\mathrm{H}$. pylori in the population under study and according to other studies, life style change and healthy nutrition are considered as effective factors in PUD prevention. According to the results of this study, the patients with upper GI symptoms should be suspect to PUD and positive H. pylori infection.
\end{abstract}

Keywords: Endoscopy; Peptic Ulcers; Helicobacter

\section{Background}

Life style change is considered as a factor affecting on the increasing number of subjects with peptic ulcer disease (PUD) (1). PUD incidence in different populations has been measured $0.03 \%$ - $0.19 \%$ through a year. Synchronic studies have shown reduction of PUD during the recent decades. The two most common causes of PUD are infection with Helicobacter pylori (H. pylori) bacterium and use of NSAIDs (1). H. pylori, is one of the most common bacterial infections across the world and at least $50 \%$ of the world's population suffers from it (2). More than 2.3 of the affected population live in developed countries.

H. pylori is a gram-negative, spiral and microaerophilic bacterium that causes gastritis and it may lead to pyloric and intestinal ulcers, iron and vitamin B12 deficiencies, gastric adenocarcinoma and primary B- cell lymphoma
(3). H. pylori is recognized as the most common cause of gastritis and also as one of the most important factors which develop gastrointestinal complications as well as indigestion symptoms $(2,4)$. It is essential to eliminate H. pylori completely, if we want to treat the related PUD (5), reduce gastrointestinal ulcer recurrence and prevent gastrointestinal ulcer in patients who should take NSAIDs (6).

Clinical symptoms are not the only factor for the exact diagnosis of PUD and in this regard endoscopic evaluation is needed (7). It should be mentioned that endoscopy is routinely suggested to evaluate gastrointestinal symptoms (8). H. pylori diagnostic tests may be divided in two categories: endoscopic and non- endoscopic. While endoscopy procedure, rapid urease test (RUT) is considered

Implication for health policy/practice/research/medical education:

The prevalence of PUD is more common in asymptomatic males than females. Regarding the high prevalence of PUD and $H$. Pylori in the population under study, life style change and healthy nutrition are considered as effective factors in PUD prevention. According to the results of this study, the patients with upper GI symptoms should be suspect to PUD and positive H. Pylori infection.

Copyright (c) 2014, Razavi Hospital. This is an open-access article distributed under the terms of the Creative Commons Attribution License, which permits unrestricted use, distribution, and reproduction in any medium, provided the original work is properly cited. 
as the first choice. Other tests include histologic evaluation, culture and PCR (2).

There is no need for the accurate estimation of PUD prevalence in general population in order to evaluate the effect of risk factors on public health or preventive interventions. Rate of PUD prevalence is useful in research studies and also health authorities (9).

\section{Objectives}

This study aimed to analyze the prevalence of PUD and H. pylori in asymptomatic subjects referring to the checkup clinic of Razavi Hospital and the relation between PUD and $H$. pylori. In addition the study is useful in determining the reference levels for health decision making.

\section{Materials and Methods}

In this is a cross-sectional study, 499 subjects who had referred to the check-up clinic of Razavi Hospital from 2006 to 2011 were included. Their demographic data, endoscopy report and rapid urease test (RUT) were evaluated. All of the mentioned subjects were asymptomatic. After collecting demographic data and filling the consent form, endoscopy and RUT test were performed for all the patients. 260 patients were prepared for the biopsy test. The results were registered and analyzed by SPSS and the study goals were evaluated by probability analysis including chi square test. All the patients, for whom upper Gastrointestinal (GI) endoscopy and also RUT test had been performed included in the study and those who did not perform endoscopy and RUT test for whatever reason, were excluded.

According to endoscopy findings, the clinical characteristics of PUD included: sub epithelial hemorrhage, gastritis, ulcer, erosion, erythema, gastroduodenitis, and duodenitis. According to biopsy findings, the clinical characteristics of PUD include gastritis and duodenitis.

\section{Results}

The mean age of the subjects was $51.7 \pm 12.18$ which included $73.1 \%$ males and $26.9 \%$ females. $70 \%$ of the subjects who did RUT were reported with positive RUT and $30 \%$ with negative RUT. After endoscopy, it was revealed that $66.8 \%$ of the subjects with positive RUT had PUD and only $9 \%$ showed no PUD. According to analyzed data, there was a significant relationship between endoscopy results and RUT $(\mathrm{P}=0.001)$.

Positive H. pylori were reported in $75.2 \%$ of males and $24.8 \%$ of females. Endoscopy results revealed $57.2 \%$ subjects with PUD from which $23 \%$ were seen with erosin, $11.6 \%$ with gastritis, $11.4 \%$ with ulcer, $8 \%$ with erythema, $1.6 \%$ with sub epithelial hemorrhage, $0.8 \%$ with duodenitis and $0.8 \%$ with gastroduodenitis (Table 1 ).

In $72 \%$ of subjects that their endoscopy showed PUD and in $38 \%$ of subjects with normal endoscopy, positive RUT was reported. In $89.6 \%$ of subjects who had PUD in biopsy, $57.7 \%$ subjects had gastritis with positive $H$. pylori and $13.8 \%$ subjects had gastritis without $H$. pylori (Table 2).

Table 1. The Relationship Between Gastrointestinal Endoscopy Findings and RUT

\begin{tabular}{|c|c|c|c|}
\hline Characteristics & Frequency & Positive RUT $^{a}$ & Negative RUT \\
\hline Normal & $90(18 \%)$ & 33 & 52 \\
\hline Erythema & $40(8 \%)$ & 30 & 8 \\
\hline Erosion & $115(23 \%)$ & 89 & 22 \\
\hline Ulcer & $57(11.4 \%)$ & 45 & 11 \\
\hline Polyp & $12(2.4 \%)$ & 8 & 4 \\
\hline GERD & $4(0.4 \%)$ & 0 & 2 \\
\hline Esophagitis & $72(14.4 \%)$ & 44 & 22 \\
\hline Hiatus Hernia & $28(5.6 \%)$ & 19 & 8 \\
\hline Esophageal varices & $1(0.2 \%)$ & 1 & 0 \\
\hline Gastritis & $58(11.6 \%)$ & 45 & 10 \\
\hline Subepithelial hemorrhage & $8(1.6 \%)$ & 8 & 0 \\
\hline Nodularity & $5(1 \%)$ & 4 & 1 \\
\hline Atrophy & $2(0.4 \%)$ & 1 & 1 \\
\hline Irregularity & $1(0.2 \%)$ & 1 & 0 \\
\hline Duodenitis & $4(0.8 \%)$ & 4 & 0 \\
\hline Gastroduodenitis & $4(0.8 \%)$ & 3 & 1 \\
\hline
\end{tabular}


Table 2. Frequency of Gastrointestinal Lesions Based on the Biopsy Results

\begin{tabular}{ll}
\hline Characteristics & Frequency \\
\hline Reflux esophagitis & $7(1.4 \%)$ \\
\hline Nonspecific esophagitis & $7(1.4 \%)$ \\
\hline Esophagitis & $6(1.2 \%)$ \\
\hline Nonspecific gastritis without $\boldsymbol{H}$.pylori & $36(7.2 \%)$ \\
\hline Nonspecific gastritis with $\boldsymbol{H}$.pylori & $87(17.4 \%)$ \\
\hline Follicular gastritis with $\boldsymbol{H}$.pylori & $38(7.6 \%)$ \\
\hline Intestinal metaplasia/dysplasia with H.pylori & $67(13.4 \%)$ \\
\hline Atrophic gastritis with $\boldsymbol{H}$.pylori & $4(0.8 \%)$ \\
\hline Nonspecific gastritis & $1(0.2 \%)$ \\
\hline Nonspecific duodenitis & $7(1.4 \%)$ \\
\hline
\end{tabular}

According to biopsy results, there was a significant relation between PUD prevalence and H. pylori $(\mathrm{P}=0.001)$. From 260 subjects who had been prepared for biopsy, 82\% were reported with RUT. Accurate analysis of data showed that there was a significant relation between gastritis incidence based on biopsy and RUT, so that $83 \%$ of the subjects were reported with positive RUT. In addition there was a significant relation between gastritis with and without $H$. pylori separately and RUT results $(\mathrm{P}=0.001)$.

\section{Discussion}

According to this study and other achieved studies, the prevalence of $H$. pylori infection and also PUD are more common in males than females $(4,8,10,11)$.

Among all subjects, 70\% who did endoscopy and RUT, were reported with positive $H$. pylori. According to the study achieved in Thailand and China, the prevalence of $H$. pylori has been reported $65 \%$ and $73.3 \%$ respectively. Prevalence of this bacterium in northwestern and central parts of Iran has been $45.3 \%$ and $89.2 \%$ respectively $(7,10)$. Since Iran is a vast country, the prevalence rate of $H$. pylori differs in various areas. Prevalence of this bacterium is more common in developing countries than developed ones. Geographic differences impact on $H$. pylori prevalence too (4).

According to endoscopy results, the prevalence of PUD in the whole population under study was reported $57.2 \%$ and in subjects with positive $H$. pylori was $66.8 \%$. Due to the achieved studies in northwestern and central parts of Iran, PUD prevalence has been $8.2 \%$ and $14.1 \%$ respectively. $(7,10)$ This prevalence was reported $32.7 \%$ in China (12).

Among the subjects, $72 \%$ whose endoscopy results showed PUD and 38\% of those with normal endoscopy were reported with $H$. pylori infection based on RUT. Rate of the prevalence in Chinese subjects with PUD was 92.6\% (12). According to endoscopy findings, the most common complications of PUD include erosion (23\%), gastritis, ulcer and erythema.

In this study after taking biopsies from the subjects,
$89.6 \%$ of them were reported with PUD from which $82 \%$ had positive RUT. A significant relation was revealed between PUD and H. pylori infection. PUD affects the quality as well as health aspects of life. It will also cost people and healthcare systems (13). Due to the high prevalence of PUD and H. pylori infection in the asymptomatic population under study and also regarding the endoscopy results; life style change and healthy nutrition are considered as effective factors in PUD prevention. Observing the mentioned items, there is no need for the patient to stay long in the hospital.

Regarding the results of the study, those patients with upper GI symptoms, who are living in Mashhad, should be suspected to PUD and positive $H$. pylori infection and accordingly, medical procedure has to be specified for them.

\section{Acknowledgements}

The authors thank the Education and Research Department and clinic checkup of Razavi Hospital.

\section{Authors' Contribution}

Ali Abdolhosseini and Kambiz Totonchi saraf: doing and supervision of the study. Azra Izanloo: analysis and writing the manuscript. Najme Jafari: collecting the data.

\section{Financial Disclosure}

The authors declare no conflict of interest.

\section{Funding Support}

The Education and Research Department of Razavi Hospital provided practical support for the survey processes.

\section{References}

1. Schottker B, Adamu MA, Weck MN, Brenner H. Helicobacter pylori infection is strongly associated with gastric and duodenal ulcers in a large prospective study. Clin Gastroenterol Hepatol. 2012;10(5):487-93 e1.

2. Tsang TK, Shrestha MP. Helicobacter Pylori Infection in Peptic Ulcer Disease. In: Jianyuan C editor. Pept Ulcer Dis.; 2011. p. 39.

3. Zali MR. Facing resistance of H. pylori infection. Gastroenterol Hepatol bed bench. 2010;4(1):3-11.

4. Khalifa MM, Sharaf RR, Aziz RK. Helicobacter pylori: a poor man's gut pathogen? Gut Pathog. 2010;2(1):2.

5. Kim JJ, Kim N, Lee BH, Kang JM, Seo P, Lim MK, et al. [Risk factors for development and recurrence of peptic ulcer disease]. Korean J Gastroenterol. 2010;56(4):220-8.

6. Venerito M, Malfertheiner P. Helicobacter.Hoboken, New Jersey: Blackwell Publishing Ltd; 2010.

7. Barazandeh F, Yazdanbod A, Pourfarzi F, Ghajarieh Sepanlou S Derakhshan $\mathrm{MH}$, Malekzadeh R. Epidemiology of Peptic Ulcer Disease: Endoscopic Results of a Systematic Investigation in Iran. MEJDD. 2012;4(2):90-6.

8. Lieberman D, Fennerty MB, Morris CD, Holub J, Eisen G, Sonnenberg A. Endoscopic evaluation of patients with dyspepsia: results from the national endoscopic data repository. Gastroenterology. 2004;127(4):1067-75.

9. Lin KJ, Garcia Rodriguez LA, Hernandez-Diaz S. Systematic review of peptic ulcer disease incidence rates: do studies without vali- 
dation provide reliable estimates? Pharmacoepidemiol Drug Saf. 2011;20(7):718-28.

10. Fakhrieh S, Ehsani Ardakani MJ, Mohaghegh Shalmani H. Endoscopic evaluation of patients with dyspepsia: results from the large endoscopic data. Gastroenterol Hepatol bed bench. 2009;1(1):25-31.

11. Kalantari H, Nourian M. Prevalance of Peptic Ulcer Versus Non Ulcer Dyspepsia in Patients which were admitted for Endoscopy.
J Isfahan Med School. 2011;28:1304-8.

12. Li Z, Zou D, Ma X, Chen J, Shi X, Gong Y, et al. Epidemiology of peptic ulcer disease: endoscopic results of the systematic investigation of gastrointestinal disease in China. Am J Gastroenterol. 2010;105(12):2570-7.

13. Barkun A, Leontiadis G. Systematic review of the symptom burden, quality of life impairment and costs associated with peptic ulcer disease. Am J Med. 2010;123(4):358-66 e2. 